\title{
Overload Screening of Transmission Systems Using Neural Networks
}

\author{
J. Riquelme ${ }^{1}$, A. Gómez ${ }^{1}$, J.L. Martínez ${ }^{1}$ and J.A. Peças Lopes ${ }^{2}$ \\ 1 Department of Electrical Engineering, University of Seville \\ Escuela Superior de Ingenieros \\ Camino de los Descubrimientos, $\mathrm{s} / \mathrm{n}$ \\ 41092 Seville, Spain \\ 2 INESC, Instituto de Engenharia de Sistemas e Computadores \\ Largo Mompilher 22 \\ 4433 Porto, Portugal
}

\begin{abstract}
The process of determining whether a power system is in a secure or insecure state is a crucial task which must be addressed on-line in any Energy Management System. In this paper, an Artificial Neural Network, capable of accurately identifying the set of harmful contingencies, is presented, along with several results obtained from a real-size power network. The proposed approach makes use of classical numerical techniques to compensate the ANN's inputs so that it can deal with topological changes in the power system.
\end{abstract}

\section{Introduction}

A reliable power system should be operated in a "secure" state, which implies that the load is satisfied and no operating limits are violated under present operating conditions, but also after the occurrence of several unforeseen contingencies (e.g., outages of lines, transformers or generators)[13].

The process of determining whether the power system is secure or insecure is known as "Static Security Assessment", whose key issues are [2]:

1. First, a fast identification of the set of dangerous critical contingencies must be performed ("Contingency Selection or Screening").

2. Next, the impact of the critical contingencies on the power system must be evaluated, usually through a post-contingency "Load Flow" routine ("Contingency Analysis").

Given this information, a system operator can decide if preventive actions should be initiated to mitigate the potential problems.

A good "Contingency Selection \& Analysis" package must be computationally fast and accurate in correctly identifying the harmful contingencies, that is, quickly reducing the number of contingencies to be studied in detail by eliminating all the non-critical ones. 
Several algorithmic techniques have been proposed, most of which are currently being used in Energy Management Centers, to reduce the number of critical contingencies to be studied explicitly in real-time, the most popular being "Distribution Factors" [12, 9], "Performance Index" [6], and "Approximated Load-Flow" $[15,3,4,1]$ methods. In reference [11], a performance evaluation of several classical static security algorithms is presented.

Over the past few years, several approaches using "Artificial Neural Networks" (ANN) have been proposed as alternative methods for "Static Security Assessment" in power system management, both using supervised and unsupervised architectures $[7,10]$. However, both classical and ANN-based methods must deal with the following problems:

- The number of possible system configurations (i.e., network topologies) is large.

- The state of every system configuration is typically defined by several hundreds or thousands of line/transformer power flows, along with a similar number of electrical bus voltages.

- Each pre-contingency configuration gives rise to a huge number of postcontingency topologies.

Perhaps owing to the combinatorial explosion of the number of contingencies and variables, almost all ANN applications to "Security Assessment" reported in the literature have only been applied to small academic power systems.

In this article, an ANN intended to carry out a "Contingency Screening" for line/transformer overloads is presented and tested on the Andalusian Transmission Network, composed of about 130 electrical buses (400 and $220 \mathrm{kV}$ buses) and 250 lines and transformers.

The paper is structured as follows: First, an ANN for a specific system topology is presented, following the techniques proposed in [5]. Secondly, a hybrid approach, which makes use of an ANN along with classical numerical techniques, is also presented and tested, showing that it is capable of dealing with several pre-contingency topologies by "compensating" the input variables of the ANN.

\section{Scheme 1: ANN Based on Line Power Flows}

The power system for which the NN is intended refers to the Southern Spanish Transmission Network, comprising 256 branches (lines/transformers), 130 buses and 22 generators (these figures include an equivalent of the external national network).

The ANN adopted is of the multi-layer perceptron (MLP) type, its structure being determined by the following considerations:

- As the goal of the ANN is to detect line overloads, it seems intuitive to choose the line apparent power flows as input variables. This information is readyly available as a byproduct of the state estimator. However, in order to reduce the number of inputs as much as possible, a feature selection algorithm, 
based on the $\mathcal{F}$ index [8, chapter 5], was used to determine which of the 256 possible power flows were actually significant. As a result, only 54 branch power flows were selected.

- There is no clear guidelines to decide how many neurons should comprise the hidden layer. In our case, after several trials, it was decided that 108 hidden neurons constitute a good compromise between complexity and performance.

- Ideally, the ANN should have as many output variables as relevant outages we wish to assess. In practice, however, operators are much more interested in the consequences of outages affecting the $400 \mathrm{kV}$ transmission lines, because they carry huge amounts of power. In this case, 7 out of the $11400 \mathrm{kV}$ internal branches, plus 3 branches of the external network, were monitored. In the end, only 9 output variables were adopted, since it was noticed that two of the contingencies systematically led to identical results. The output values are constrained to the interval $[0,1]$, a value larger than 0.5 indicating that the outage associated with this particular output causes overloading in any system component.

This MLP, whose neurons make use of the sigmoid transfer function, was trained by means of the back propagation algorithm. As many as 746 network states were generated by systematically modifying the load profile and running a load flow program. Note that all situations refer to the same base-case topology. Two thirds of these states were chosen to train the NN, keeping in mind that both secure and insecure states were included in the same proportion, while the remaining subset was used in the test phase.

The performance of the MLP on these test cases was extremely good. There were no missed detections and only a single false alarm, which represents an error of $0.4 \%$ over the entire test set $(0.04 \%$ when the 10 outputs per case are considered). Since the main purpose of the ANN is to detect critical contingencies, which should be subsequently analyzed in detail by a load flow routine, the existence of a very small percentage of false alarms is not too important, because the consequence of this kind of error is merely a moderate increase in the computational cost at the contingency analysis stage.

Although most of the time all components of the transmission network are in service in order to increase the security level, it sometimes happens that a particular line or transformer is disconnected owing to periodical maintenance, etc. The ANN described above was also tested using a battery of cases in which the base-case topology was modified by disconnecting a single element. According to expectations, the percentage of misclassifications largely exceeded $30 \%$, because the NN had not been trained with the modified topologies.

Consequently, a new set of 5861 cases was created comprising 5 different topologies in addition to the base-case topology. Four of the new topologies differ from the base case in a single element, while the fifth case constitutes a rather extreme case in which 3 components are simultaneously disconnected. When $2 / 3$ of the whole set of cases are used again to train the NN, only 12 out of the 1953 test cases led to one or several classification errors, as shown in Table 1. 


\begin{tabular}{|l||cc||cc||ccc||ccccc|}
\hline Topology & \multicolumn{2}{|c||}{0} & \multicolumn{1}{|c||}{1} & & 3 & \multicolumn{7}{|c||}{5} \\
\hline Case \# & 1 & 2 & 3 & 4 & 5 & 6 & 7 & 8 & 9 & 10 & 11 & 12 \\
\hline \hline False Alarms & 1 & 1 & 1 & 1 & 1 & 2 & 1 & 0 & 0 & 2 & 1 & 1 \\
\hline Missed Detections & 0 & 0 & 1 & 1 & 0 & 0 & 0 & 6 & 6 & 0 & 0 & 0 \\
\hline
\end{tabular}

Table 1. Statistics corresponding to the 12 cases containing one or several wrong outputs.

The following comments are in order regarding these results:

- The NN gives now 2 false alarms, instead of 1, for the base-case topology (labeled 0 in Table 1). This performance deterioration is caused by the new learning process, aimed at tuning the NN to 5 additional topologies.

- The 2 missed detections corresponding to topology \#1 could have been avoided with a more conservative threshold (the value of the 2 affected outputs was 0.48 while the threshold was 0.5 ). However, this solution would lead to extra false alarms.

- As expected, the worst results were obtained with the fifth topology, which significantly departs from the base case (3 elements are disconnected). No errors appear with topologies 2 and 4.

- Overall, taking into account that each of the 1953 test cases provides information about 10 different contingencies, the 12 false alarms and the 14 missed detections summarized in Table 1 represent only $0.06 \%$ and $0.07 \%$ respectively of the whole set of NN outputs. This performance is better than the one obtained with conventional contingency screening techniques, based on distribution factors or incremental load-flow models [11].

\section{Scheme 2: ANN Based on Bus Power Injections}

Although the results presented in the previous section are promising, it should be remembered that the NN was trained by means of only 6 different topologies to detect the overloads caused by just 10 contingencies. It would be quite difficult, if not impossible, to develop a single NN capable of recognizing any conceivable network topology and providing an output for every branch and generator outage, at least for realistic networks.

In an attempt to somehow elude the dependence on network topology and on the particular contingency being studied, a NN based on bus power injections has been developed and tested. As discussed in [8, chapter 9], power flows contain implicit information on the topology of the network, but the dimension of the power injection vector is smaller and the absence of input redundancy is guaranteed.

The basic idea behind the new NN is that both what makes the current topology different from the base case and the information on the particular contingency being studied, can be approximately represented by properly modifying 
the injection vector, much in the same way as compensation techiques [14] are applied in conventional methods to deal with localized network changes.

Again, the MLP type was adopted but, according to the new operating principles, its structure is as follows:

- The output layer comprises a single neuron whose output provides the same information as before, i.e., a value higher than 0.5 indicates that at least one branch is overloaded. This means that the NN must be resorted to every time a particular contingency has to be studied, unlike in the previous scheme where a single pass provided all outputs corresponding to the reduced set of contingencies for which the NN had been trained.

- The input buffer, as stated earlier, contains as many elements as the number of buses, plus an extra element representing the total demand. The precise meaning of these inputs will be explained below.

- The number of neurons comprising the hidden layer has been chosen in acordance with the number of network branches. A similar choice was made in [8], where 36 neurons were found optimal for a 35-branch network.

This time, the transfer function adopted is the hyperbolic tangent and the learning process is based on a modification of the cumulative back propagation rule known as the "Normalized-Cumulative-Delta Learning Rule".

The values fed into the NN inputs depend on the case in hand. Both in the learning and test phases the $\mathrm{NN}$ is used as if the network topology was always that of the base case. Consequently, when we are dealing with any base-case load profile, the NN is trained and tested by simply setting every input value to the net active power injection of the respective bus, normalized with the total demand. If we are interested in the outage of a branch carrying a certain active power flow from bus $i$ to $j$, this flow must first be added to and substracted from the injection at buses $i$ and $j$ respectively, before resorting to the NN. This modification of the involved power injections is not intended to accurately compensate for the outaged branch, but as a simple means of informing the NN that there has been a change with respect to the base case affecting the two buses. When the topology of the current network state differs from that of the base case, the missing element is first identified (owing to security reasons, it is very unlikely that several components are simultaneously disconnected at the high-voltage levels). Then, a load flow program is run to find the power flows that would exist if the disconnected element was in service (the overhead caused by this numerical step is still modest compared to the cost of the overall process). From this step on, the way of proceeding is similar to the one explained above, except for the fact that the adjacent injections of both the voluntarily disconnected and the outaged branches must be properly modified.

The role played by the ANN in the contingency selection phase of the whole security assessment process is shown in figure 1.

In order to assess the efectiveness of this alternative approach, the same transmission network of section 2 has been used. In this case, the network is composed of 123 buses and 227 branches, and the total load amounts to 5711 


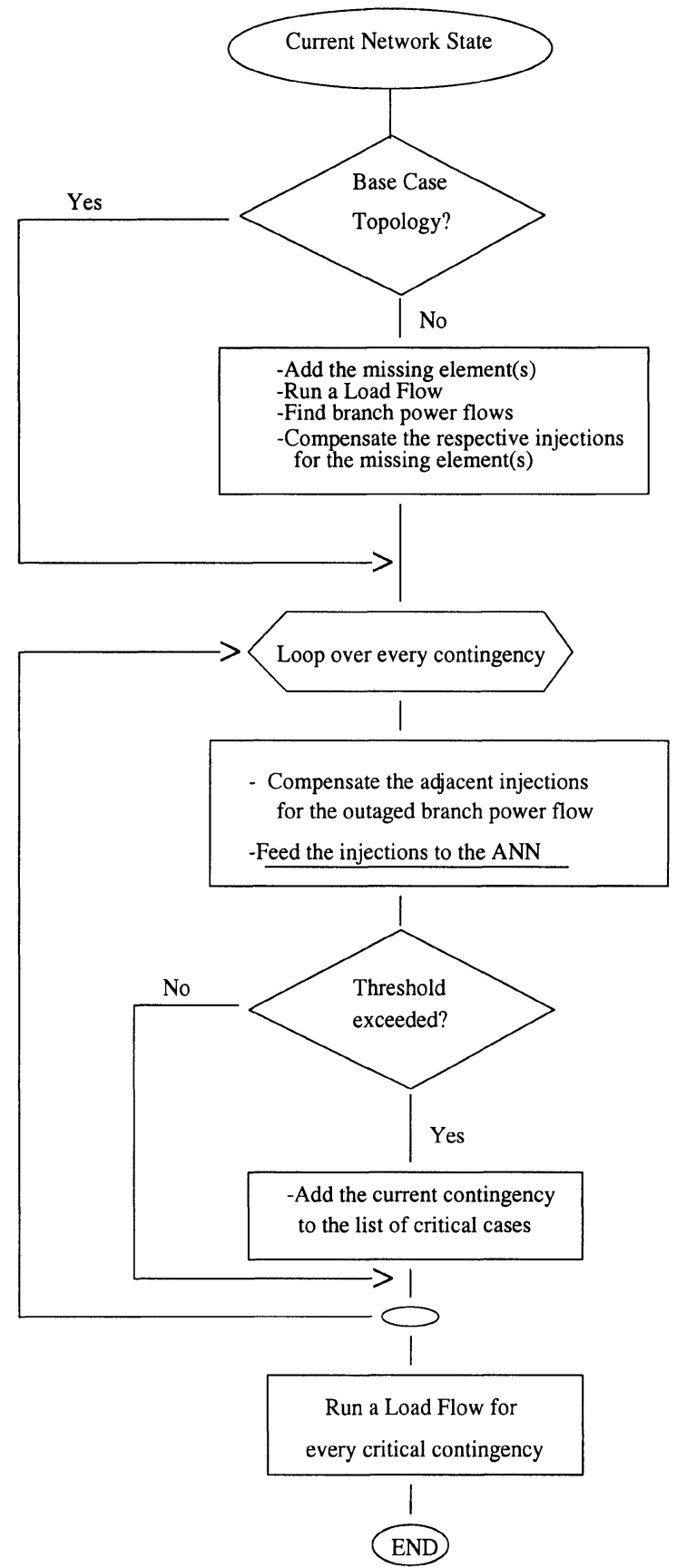

Fig. 1. Flow diagram of the hybrid security assessment approach. 
MW (this state corresponds to a snapshot saved on April 27, 1994 during the peak load situation). Excluding radial and external branches, a total of 110 contingencies are of interest (in this study, only branch outages will be considered, but generator outages could be very easily included since they do not involve topology changes). By linearly decreasing the load demand, and by resorting to an optimal power flow to find reasonable generation patterns, 18 additional states were obtained. Each of the resulting states, combined with all possible branch outages, yield a total of 2109 cases $(19 \cdot 111)$ which were used to train the NN. After the training process, that took 42500 iterations, the training set was aplied again to the NN resulting in 3 false alarmas (0.14\%) and no missed detections. This means that only 3 extra cases would have to be numerically analyzed in addition to the 327 contingencies which are truly critical. Note that all cases belonging to the training set referred to contingencies affecting the same base-case topology.

The test set was generated intentionally from 8 different topologies for which the NN had not been previously trained. These alternative topologies were obtained from the base case by disconnecting a single line or transformer. In addition, 4 different loading levels were considered for every topology. Combining the 32 resulting states with all possible contingencies, and discarding a few infeasible situations, a total of 3472 test cases were applied to the NN, of which 725 corresponded to critical contingencies. The MLP response included a single missed detection and 146 false alarms (4.2\%). The missed detection turned out to be irrelevant in practice, because the only overload detected referred to a line carrying 190.8 MW. whose rating was $189 \mathrm{MW}$. The relatively high proportion of false alarms suggests that a more accurate way of modeling topological changes should be devised. However, thanks to the use of an ANN that was just trained with the base-case topology, the load flow program had to be run only 871 times, out of the 3472 tested cases, which means a saving of $75 \%$ in the computational effort.

\section{Conclusions}

In this paper, the potential application of a MLP to the screening phase of security assessment is explored in the context of realistically sized power transmission systems. Firstly, an ANN based on a selected subset of branch power flows is developed, which is capable of simultaneously dealing with a reduced number of major contingencies. Although the performance obtained with this approach is acceptable, its generalization to all possible contingencies and network topologies is not trivial. Secondly, an alternative scheme is proposed based on bus power injections, which must be properly modified to account for topological modifications and/or outaged branches. This generalized approach has shown to perform satisfactorily so long as the input topology is close enough to the base-case topology used in the training phase, which is usually the case. Further research efforts should be directed to devise more accurate ways of compensating power injections for topological changes. 


\section{References}

1. R. Bacher and W.F. Tinney, "Faster Local Power Solutions: The Zero Mismatch Approach", IEEE Trans. Power Syst., Vol. PWRS-4, pp. 1345-1354, Nov. 1989.

2. N. Balu, T. Bertram, A. Bose, V. Brandwajn, G. Cauley, D. Curtice, A. Fouad, L. Fink, M.G. Lauby, B.F. Wollemberg and J.N. Wrubel, "On-Line Power System Security Analysis", Proceedings of the IEEE, Vol. 80, no. 2, February 1992, pp. $262-280$.

3. V. Brandwajn, "Efficient Bounding Method for Linear Contingency Analysis", IEEE Trans. Power Syst., Vol. PWRS-2, pp. 38-43, Feb. 1988.

4. V. Brandwajn and M.G. Lauby, "Complete Bounding for AC Contingency Analysis", IEEE Trans. Power Syst., Vol. PWRS-4, pp. 724-729, May 1990.

5. CIGRE Task Force 38-06-06 on Artificial Neural Network Applications for Power System. Convenor Dagmar Niebur, Vol. 1 No. 3, pp. 133-157, Dec. 1993.

6. G.C. Ejebe and B.F. Wollemberg, "Automatic Contingency Selection", IEEE Trans. Power App. Syst., Vol. PAS-98, pp. 92-104, Jan/Feb. 1979.

7. Fischl et al., "Screening Power System contingencies Using Back-propagation Trained Multiperceptron", Proc. of the 1989 ISCAS, Vol. 3, pp. 486-489, 1989.

8. Mohamed-El-Sharkawi and D. Niebur, editors, "Tutorial Course on Artificial Neural Networks with Applications to Power Systems", IEEE Catalog Number: 96 Tp 1120 .

9. W.Y. Ng, "Generalized Generation Distribution Factors for Power System Security Evaluation", IEEE Trans. Power App. Syst., Vol. PAS-100, pp. 1001-1005, Mar. 1981.

10. D. Niebur and A. Germond, "Power System Static Security Assessment Using Kohonen Neural Network Classifier", IEEE Trans. Power Syst., Vol. 7 , pp. 865872, May 1992.

11. A.P. Sakis Meliopoulos, C.S. Cheng and F. Xia, "Performance Evaluation of Static Security Analysis Methods", IEEE Trans. Power Syst., Vol. PWRS-9, pp. 1441-1449, Aug. 1994.

12. P.W. Sauer, "On the Formulation of Power Distribution Factors for Linear Load Flow Methods", IEEE Trans. Power App. Syst., Vol. PAS-100, pp. 764-770, Feb. 1981.

13. B. Stott, O. Alsac and A.J. Monticelli, "Security Analysis and Optimization", Proceedings of the IEEE, Vol. 75, no. 12, pp. 1623-1644, December 1987.

14. W. F. Tinney, "Compensation methods with with ordered triangular factorization", IEEE Trans. Power App. Syst., vol. PAS-91, pp. 123-127, Jan./Feb. 1972.

15. J. Zaborsky, F.W. Whang and Prasad, "Fast Contingency Evaluation Using Concentric Relaxation", IEEE Trans. Power App. Syst., Vol. PAS-99, pp. 28-36, Jan/Feb. 1980. 